\title{
Transferring EU-funded biotechnology research to European bioindustry
}

\section{Colm Lawler, Robert van der Meer, and Jacques Viseur}

The European Union (EU) is currently deliberating the content of, and budget for, its next scientific research and development program, Framework 5 . This will begin in 1999 and, under current proposals, life sciences research will have a budget of over 2.5 billion ECUs ( $\$ 2.8$ billion). However, the results of a recent survey' of both research groups and companies suggest that a good deal of EU-funded biotechnology may be falling down gaps in some of the most basic aspects of the technology transfer process.

The uptake of EU-funded biotechnology research by European bioindustry is a stated aim of the European Commission's 4th Framework Program (1994-1998). A key mechanism through which the EU tries to achieve this aim is the Innovation Program, a scheme established in 1995 and controlled by the Directorate for Telecommunications, Information Market and Exploitation of Research of the European Commission (DG XIII). It aims to be an interface between the market and the various EU research programs and has an overall budget of over 250 million ECUs for the period 1995-1998 (inclusive). Perhaps the most startling finding of the recent survey is that most companies-from large pharmaceutical companies to small or medium-sized enterprisesknow nothing about the Innovation Program or value it little. Of 95 European companies surveyed, $66 \%$ had not heard of the Program. Of those that had, $65 \%$ did not think it effective.

It was not as if the companies were uninterested in Europe or in biotechnology: They were all members of the European Association of BioIndustries (Brussels). Furthermore, the respondents were keen on technology transfer. Two thirds of the sample (65\%) supported European collaborations in technology transfer, and $60 \%$ supported European collaboration in research and development. There was a unanimous desire, too, among the companies to be kept informed of technical opportunities arising

Colm Lawler is project manager of BioResearch Ireland (lawlerc@biores-irl.ie); Robert van der Meer is director of the Netherlands Industrial Agricultural Biotechnology Association (niaba@xs4all.nl); and Jacques Viseur is director of Eurotop Co-operation Partners Brussels (esnba@interpac.be). from EU-funded biotechnology research. If such research is to be more fully exploited, the Innovation Program must become significantly more visible and effective to European bioindustry in the Framework 5 EU Research and Development Program.

The survey also investigated attitudes regarding technology transfer among 135 researchers (a $70 \%$ response) from 14 countries who were funded under the 4th Framework Biotechnology Program. They

\section{A recent survey suggests that a good deal of EU- funded biotechnology may be falling down gaps in some of the most basic aspects of the technology transfer process.}

clearly recognized the central role of obtaining intellectual property rights (IPR) in the results of their work: $86 \%$ considered that protecting their results was important. However, a majority of all researchers $(56 \%)$ stated that they needed technical assistance in IPR, suggesting that many European universities and research organizations are falling short of researcher expectations and needs. One way to deal with this would be to establish a concerted European action/network to offer institutions-especially those without licensing departments-assistance on various aspects on IPR and technology transfer such as technology evaluation to company negotiations. One obvious model for such an umbrella association is the Association of University Technology Managers, which assists universities in transferring their technologies to the marketplace.

Although European Union-funded researchers are clearly aware of the importance of IPR, many of them have "traditional" academic attitudes to interactions with industry. For instance, they prefer to bring their results to the attention of industry through refereed journals and conference proceedings. Fifty-eight percent were in favor of an alternative form of publication- a "technology opportunity release"-but $82 \%$ of that group said they would require the assistance of an intermediary to help prepare them. The extensive use of electronic communication by $\mathrm{EU}$ researchers would make it the ideal route for informing European bioindustry of opportunities arising from EU-funded biotechnology research, and its increased use for this purpose should be considered.

The survey has highlighted that IPR assistance to European biotechnology researchers and an efficient communication interface to bring all EU-funded biotechnology research to the attention of European bioindustry are still needed. The establishment/involvement of trans-European industrial networks and associations are crucial for both tasks to be realized and could, for example, include the various Industrial Platforms established under the current Biotechnology Program. The distribution of research/technology opportunities should be targeted to companies thought most likely to take up this technology, and the major criteria covered should be those highlighted by industry such as IPR position and market applications. It is possible that the distribution network could integrate electronic communication systems in the future once it has been totally integrated by industry. Such a technology transfer network may well need intermediaries who can understand and balance the dual requirements of industry and academia.

In summary, a sound governmental support framework on a European basis is necessary to reinforce the technology transfer process between EU-funded biotechnology researchers and bioindustry. Adding value to basic research by converting discoveries into inventions and ultimately products is a process that evolves in a more targeted manner. By facilitating the access to European bioindustry of new scientific technologies arising from EU-funded biotechnology research, the competitiveness of Europe in sectors such as human health, agriculture, and environmental protection will ultimately be increased.

1. Lawier, C., van der Meer, R., and Viseur, J. May 15,1998 . The survey was $50 \%$ financed by the European Commission under the 4th Framework Biotechnology Program, 2nd Call. Contract No. ERBIO4 CT 960387 . The authors acknowledge the support of the European Association of Biolndustries (EuropaBio) in conducting this survey. 